\title{
The timing of amniotomy, oxytocin and neuraxial analgesia and its association with labour duration and mode of birth
}

\author{
Mechthild M. Gross • Cornelia Frömke • \\ Hartmut Hecker
}

Received: 8 August 2012/Accepted: 3 June 2013/Published online: 18 June 2013

(c) Springer-Verlag Berlin Heidelberg 2013

\begin{abstract}
Purpose The objective was to study the association of different timings of intrapartum interventions with labour duration and mode of birth.

Methods A longitudinal cohort study of 2,090 nulliparae and 1,873 multiparae with a singleton in cephalic presentation was conducted. We assessed the association between, on the one hand, the timing of augmentation with oxytocin, neuraxial analgesia and amniotomy, and, on the other hand, the time to complete dilatation, spontaneous or operative vaginal delivery or caesarean delivery, using a Cox regression model accounting for standard confounders.

Results From amniotomy onwards labour was accelerated. In multiparae, amniotomy was associated with an initial 6.6-fold acceleration, decreasing first stage duration until the hazard ratio reached around 3.5, where the intervention was performed $5 \mathrm{~h}$ after labour onset; thereafter, acceleration continued with a hazard ratio of around 3. In nulliparae, neuraxial analgesia was associated with a shorter first stage when administered between 7 and $11 \mathrm{~h}$ after labour onset; the later it was performed, the less likely was spontaneous birth and the more likely an operative vaginal birth in nulliparae or a caesarean section in multiparae. The start of oxytocin augmentation was associated
\end{abstract}

\footnotetext{
M. M. Gross $(\square)$ · C. Frömke

Midwifery Research and Education Unit,

Department of Obstetrics, Gynaecology and Reproductive

Medicine, Hannover Medical School, Carl-Neuberg-Str. 1, 30625 Hannover, Germany

e-mail: Gross.Mechthild@mh-hannover.de

URL: www.mh-hannover.de/Hebammenwissenschaft.html

H. Hecker

Department of Biometry, Hannover Medical School, Carl-Neuberg-Str. 1, 30625 Hannover, Germany
}

with acceleration towards both full dilatation and caesarean section during first stage and an increased risk of operative vaginal birth during second stage. The later oxytocin augmentation started, the more likely it was that spontaneous birth would be retarded in multiparous women. Conclusions Applying amniotomy, oxytocin and neuraxial analgesia at their optimal timing may improve the progress and outcome of labour.

Keywords Amniotomy - Augmentation with oxytocin . Labour duration $\cdot$ Neuraxial analgesia $\cdot$ Time-to-event analysis

\section{Introduction}

Intrapartum interventions are intended to improve the progress of labour. While their impact on mode of birth has been analysed for decades [1], the timing of intrapartum interventions has been studied only in terms of early or late application [2, 3].

With regard to neuraxial analgesia, there has recently been a shift towards a more individual approach that takes the wishes of the mother into account [4]. Early neuraxial analgesia does not increase the risk of caesarean delivery and is able to shorten the first stage [2, 4-6]. There are no longitudinal studies examining whether its effect is constant over time or varies with timing, or how these effects might be related to the risk of a caesarean section being required.

Intravenous oxytocin augmentation is usually administered to expedite labour. Until now, oxytocin has rarely been assessed as an isolated intervention in randomized trials [7, 8]. Oxytocin administration is associated with a significantly higher risk of an instrumental delivery or an 
emergency caesarean $[8,9]$. No data on how these outcomes are related to the timing of oxytocin administration is yet available. Oxytocin is also widely used to compensate for the slowing effect of neuraxial analgesia during labour $[9,10]$ or after either spontaneous or artificial rupture of the membranes. According to a Cochrane review, amniotomy does not produce any statistical difference in the length of the first stage of labour [11]. It may reduce the incidence of dysfunctional labour, but there is no definitive information on whether this effect is associated with its timing [12].

While most of these results were obtained from randomized trials and systematic reviews, little is known about how the timing of amniotomy, neuraxial analgesia and oxytocin impacts on the process and outcome of labour [13]. The methodological challenges of examining labour progression have more recently been appreciated, especially in relation to clinical practise [14-17]. Observational studies have suggested that time-constant factors such as increased birth weight [18] or maternal age [19] are associated with duration of labour and non-spontaneous modes of birth. It remains uncertain how the timing of intrapartum interventions is associated with length of labour and mode of birth.

The aim of this paper is to study the associations between the timing of augmentation with oxytocin, amniotomy and neuraxial analgesia, as well as confounders, and the duration and mode of birth.

\section{Methods}

Data for this longitudinal cohort study in the German state of Lower Saxony were collected in 47 maternity units during and after births between April and October 2005 [12]. Pregnant women bearing a single viable foetus in cephalic presentation and planning a vaginal birth were eligible for the study. As recommended care for preterm labour differs from that for term labour [20], the study was limited to pregnancies of at least 34 completed weeks. Usually, the midwife on duty in the labour ward determines the time of the onset of labour retrospectively by reference to the occurrence of regular or irregular contractions, occasionally accompanied by ruptured membranes. The onset of labour was defined as regular or irregular contractions in association with increasing cervical dilatation which was assessed by the midwife. Institutional approval for the anonymous gathering of information was granted by the Ethics Committee of Hannover Medical School and by the Ethics Committee for public hospitals.

To account for possible confounders which might be associated with labour duration or mode of birth [18, 19], additional variables were included in the model. These were grouped as demographic, risk-associated, induction and infant variables. They were described as time-constant variables, since for the individual woman these factors did not change during labour. Women with a previous caesarean section but no vaginal birth were classified as nulliparae. In Table 1, a descriptive analysis for durations is presented.

Time-dependent intrapartum factors were amniotomy, start of neuraxial analgesia [21] and start of oxytocin augmentation. Midwives are the main care provider during the process of labour. If they determine that analgesia during labour is indicated, they will suggest this to the physician. There is no routine vaginal examination ahead of an intervention [12]. Epidural catheter technique was used in 18 units [21]; combined spinal-epidural methods were used only in one unit. Most of the units used bolus injection $(n=42)$.

The time-dependence of these interventions was modelled as an indicator function, defined on a time axis after onset of labour and switching from 0 to 1 at the time of the intervention. With respect to time-to-event data, full cervical dilatation and spontaneous birth were taken as the events concluding the first and second stages, respectively. Caesarean during first or second stage and operative vaginal birth were regarded as alternative endpoints. As all these events are regarded as competing risks in the analysis, no censoring was necessary.

Time-to-event analysis was applied as a generalization of Cox regression to examine associations between timedependent and time-constant factors and the competing risks of the various endpoints [22]. In this approach, throughout the birth process the possible transitions into one of the endpoints under consideration (i.e. spontaneous birth, caesarean section or operative vaginal birth) were modelled as time-related transition probabilities or "hazards". These hazards may vary, depending on the values of their time-dependent or time-constant covariates. The change in the hazards resulting from covariates is measured as the hazard ratio (HR), which denotes the relative change in the hazard if the covariate is changed by +1 in the units of the covariate scale. Thus, HR $=2$ indicates a doubling of the hazard for the specified endpoint when the value of the covariate changes from 0 to 1 .

The study was designed to detect a hazard ratio of 1.2 at the level alpha (two-sided) $=0.05$ with a power of $80 \%$. Therefore, the necessary number of events was calculated at 944. It was intended to achieve the same power for events of a $50 \%$ probability. Thus the target number of labours to be analysed among nulliparae and parae was $n=1,888$ in each case.

Variable selection for time-constant factors was performed in a two-stage procedure. Firstly, first and second stages were analysed separately without the time-dependent 
Table 1 Description of variables

Nulliparae $n=2,090$

Time-constant factors

Demographic factors

Maternal age in years-Md (min-max)

Parity $(2[=$ reference $]$ vs. $3 ;>4)\{n(\%)\}$

Risk-associated factors

Difference from EDD in days-Md (min-max)

No risks in medical history $\{n(\%)\}$

No prolonged pushing due to previous disease $\{n(\%)\}^{\mathrm{a}}$

Diabetes mellitus $\{n(\%)\}$

Smoking $\{n(\%)\}$

Obesity $\{n(\%)\}$

VBAC status $\{n(\%)\}$

Spontaneous rupture of membranes before onset of labour $\{n(\%)\}$, md in hours (min in min-max in days)

Meconium-stained liquor before onset of labour $\{n(\%)\}$

Foetus/infant

Birth weight in gram $-\mathrm{md}(\min -\max )^{2}$

Length in $\mathrm{cm}-\mathrm{md}(\min -\max )^{2}$

Head circumference in $\mathrm{cm}-\mathrm{md}(\min -\max )^{2}$

Sex female (reference $=$ male) $\{n(\%)\}^{\mathrm{b}}$

Induction

Overall $\{n(\%)\}$

Prostaglandin $\{n(\%)\}$

Oxytocin $\{n(\%)\}^{\mathrm{c}}$

Castor oil $\{n(\%)\}$

Misoprostol $\{n(\%)\}$

Amniotomy $\{n(\%)\}^{\mathrm{c}}$

Time-dependent intrapartum factors

Amniotomy $\{n(\%)\}^{\mathrm{d}}$

Onset of labour until amniotomy in hours - $\mathrm{md}(\mathrm{min} / \mathrm{max})$

Amniotomy until birth in hours-md ( $\min / \max )$

Oxytocin $\{n(\%)\}^{\mathrm{d}}$

Onset of labour until oxytocin in hours-md ( $\min / \mathrm{max})$

Oxytocin until birth in hours-md $(\mathrm{min} / \mathrm{max})$

Epidural $\{n(\%)\}$

Onset of labour until epidural in hours-md $(\mathrm{min} / \mathrm{max})$

Epidural until birth in hours-md ( $\min / \max )$

Endpoints

Duration of labour in vaginal births ${ }^{\mathrm{e}}$

First stage (hours) $-\mathrm{md}(\min / \max )^{\mathrm{f}}$

Second stage (hours) - $\operatorname{md}(\min / \max )^{\mathrm{g}}$

Labour duration (hours) - $\operatorname{md}(\min / \max )^{\mathrm{e}}$

Mode of birth

Spontaneous

Operative vaginal birth
$28(14-46)$
n/a

$0(-42,+14)$
$1,169(55.9)$
$12(0.6)$
$13(0.6)$
$117(5.6)$
$83(3.8)$
$211(10.1)$

531 (25.4), 6.5 (2 min-91.94 d)

$30(1.4)$

$3,420(1,720-5,370)$

$52(40-60)$

$35(25.5-41)$

$1,065(51.0)$

$612(29.3)$

346 (16.6)

$138(6.6)$

$150(7.2)$

$93(4.5)$

$23(1.1)$

$718(34.4)$

$5.73(0.00 / 74.92)$

$2.3(0.67 / 9.90)$

$1,096(52.44)$

$6.00(0.0 / 54.42)$

$3.17(0.05 / 17.12)$

$727(34.8)$

$4.5(-5.78 / 51.17)$

$5.18(0.27 / 14.55)$

$7.75(0.17 / 75.3)$

$0.75(0.03 / 5.02)$

$8.58(0.53 / 75.60)$

1,497 (71.6)

$216(10.3)$
Multiparae $n=1,873$

\author{
$31.5(18-46)$ \\ $1,149(61.3)$ \\ $484(25.8)$ \\ $210(12.8)$
}

$-0.5(-40,+18)$

1,007 (53.8)

$6(0.3)$

$18(1.0)$

$137(7.3)$

$100(5.3)$

$91(0.5)$

296 (15.8), 4.25 (5 min-7.75 d)

$14(0.7)$

$3,530(1,760-5,240)$

52 (38-63)

35 (27-39.5)

952 (50.9)

460 (24.6)

238 (11.4)

$146(7.8)$

138 (7.4)

$62(3.0)$

22 (1.2)

$782(41.8)$

$3.67(0.00 / 98.00)$

$0.6(0.02 / 8.38)$

505 (26.96)

$4.08(0.0 / 98.17)$

$1.42(0.02 / 16.67)$

233 (12.4)

$3.17(-4.2 / 28.55)$

$2.83(0.25 / 10.27)$

$4.17(0.08,101.6)$

$0.20(0.02 / 3.63)$

$4.50(0.05 / 101.65)$

$1,764(94.2)$

31 (1.7) 
Table 1 continued

\begin{tabular}{lll}
\hline & Nulliparae $n=2,090$ & Multiparae $n=1,873$ \\
\hline Caesarean section during first stage & $296(14.2)$ & $65(3.4)$ \\
Caesarean section during second stage & $81(3.9)$ & $13(0.8)$ \\
Retrospective documentation (ref. = prospective) $\{\mathrm{n}(\%)\}$ & $1,480(70.8)$ & $1,314(70.2)$ \\
\hline
\end{tabular}

Md median, EDD estimated due date, $V B A C$ vaginal birth after caesarean, $d$ day, $n / a$ not applicable, max maximum, min minimum, $r e f$. reference

${ }^{\text {a }}$ No. of Nulliparae (Np)/Multiparae (Mp): $n=2,090 / n=1,872$

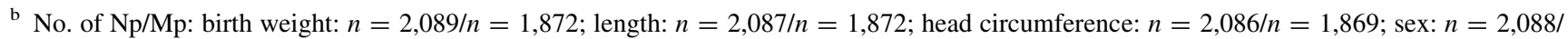
$n=1,872$

c Administered before onset of labour

d Administered at/after onset of labour

e $n=593 \mathrm{~Np} / n=109 \mathrm{Mp}$ censored due to operative vaginal delivery or caesarean section. Cases with negative or missing times are ignored with Kaplan-Meier estimation ( $n=3 \mathrm{Mp})$

${ }^{\mathrm{f}} n=593 \mathrm{~Np} / n=109 \mathrm{Mp}$ censored due to caesarean section. Cases with negative or missing times are ignored with Kaplan-Meier estimation $(n=3 \mathrm{Mp})$

g $n=297 \mathrm{~Np} / n=47 \mathrm{Mp}$ censored due to operative vaginal delivery or caesarean section. Cases with negative or missing times are ignored with Kaplan-Meier estimation $(n=296 \mathrm{~Np}, n=65 \mathrm{Mp})$

Table 2 Generalized Cox regression model after backward selection by stage of labour

\begin{tabular}{|c|c|c|c|c|c|c|c|}
\hline \multirow[t]{2}{*}{ Row } & \multirow[t]{2}{*}{ Start of episode } & \multirow[t]{2}{*}{ End of episode } & \multirow[t]{2}{*}{ Variable } & \multicolumn{2}{|c|}{ Nulliparae } & \multicolumn{2}{|c|}{ Multiparae } \\
\hline & & & & HR & CI $95 \%$ & HR & CI $95 \%$ \\
\hline 1 & Labour onset & Full dilatation & Intrapartum amniotomy & 2.73 & $2.43-3.06$ & 6.61 & $4.66-9.37$ \\
\hline 2 & & & Intrapartum amniotomy $*$ time & & & 0.71 & $0.59-0.88$ \\
\hline 3 & & & Intrapartum oxytocin $*$ time & 1.28 & $1.21-1.35$ & 1.15 & $1.07-1.23$ \\
\hline 4 & & & Neuraxial analgesia & 0.52 & $0.32-0.83$ & 0.85 & $0.73-0.99$ \\
\hline 5 & & & Neuraxial analgesia $*$ time & 1.38 & $1.11-1.72$ & & \\
\hline 6 & Labour onset & CS during first stage & Intrapartum amniotomy & 1.60 & $1.21-2.18$ & & \\
\hline 7 & & & Intrapartum oxytocin & 2.21 & $1.65-2.97$ & & \\
\hline 8 & & & Intrapartum oxytocin $*$ time & & & 1.69 & $1.27-2.24$ \\
\hline 9 & & & Neuraxial analgesia $*$ time & 1.37 & $1.22-1.55$ & 1.46 & $1.09-1.94$ \\
\hline 10 & Full dilatation & Spontaneous birth & Intrapartum amniotomy & 1.19 & $1.07-1.33$ & 2.23 & $1.59-3.13$ \\
\hline 11 & & & Intrapartum amniotomy $*$ time & & & 0.72 & $0.59-0.87$ \\
\hline 12 & & & Intrapartum oxytocin $*$ time & & & 0.90 & $0.85-0.96$ \\
\hline 13 & & & Neuraxial analgesia & 0.46 & $0.41-0.52$ & & \\
\hline 14 & & & Neuraxial analgesia $*$ time & & & 0.71 & $0.65-0.77$ \\
\hline 15 & Full dilatation & Operative vaginal birth & Intrapartum amniotomy & & & 0.43 & $0.19-0.99$ \\
\hline 16 & & & Intrapartum oxytocin & 2.46 & $1.73-3.50$ & 2.90 & $1.33-6.33$ \\
\hline 17 & & & Neuraxial analgesia & 0.08 & $0.01-0.43$ & & \\
\hline 18 & & & Neuraxial analgesia $*$ time & 2.84 & $1.37-5.89$ & & \\
\hline 19 & Full dilatation & CS during 2nd stage & Intrapartum oxytocin & 2.52 & $1.39-4.59$ & & \\
\hline 20 & & & Neuraxial analgesia $*$ time & & & 2.70 & $1.40-5.21$ \\
\hline
\end{tabular}

Adjusted for time-constant factors

variables, using common Cox regression. For each domain (e.g. the demographic) the variables were analysed to screen for factors that were significant on a multivariate level within their domain. Secondly, the significant time-constant factors and the three time-dependent variables were included in the generalized Cox regression model. In the model all variables were included in a linear additive manner.
Interactions were included for the time-dependent variables only. A backward variable selection procedure using a cutoff level for inclusion of $\alpha=5 \%$ was applied here too. The final models are presented in Table 2.

For the statistical analysis the software packages SPSS Version 14.0 [23] and TDA [24] were used. R24 was used for the graphic demonstration of the results [25]. 


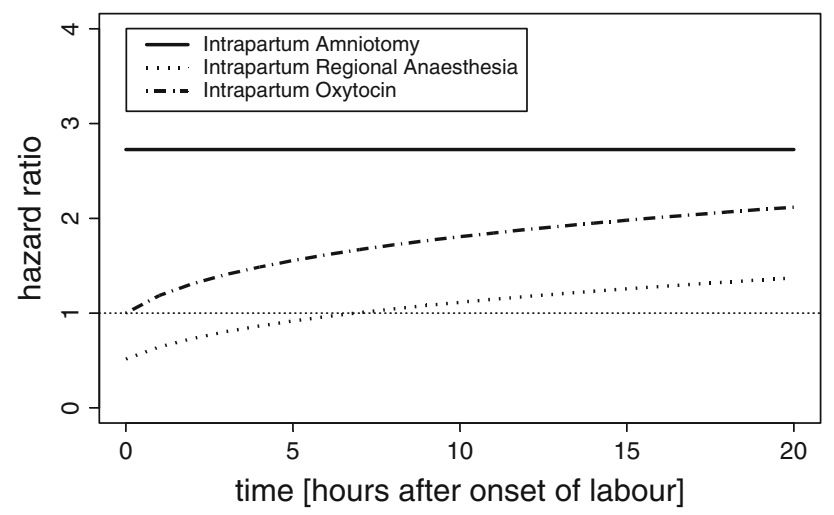

Fig. 1 Plotted graph of the effects of amniotomy, oxytocin and neuraxial analgesia on first stage duration (onset of labour-full dilatation) in nulliparae. Given that the individual has not reached the specific endpoint, a hazard ratio $>1$ at a certain time $t$ indicates an immediately increased relative risk of a transition to the specified endpoint. Estimates are adjusted for confounders, see Table 2 for detailed results

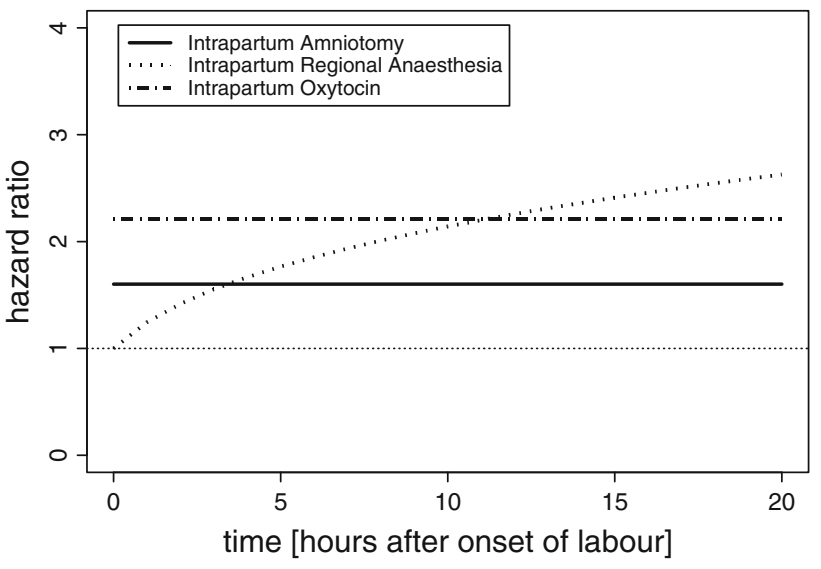

Fig. 2 Plotted graph of the effects of amniotomy, oxytocin and neuraxial analgesia on caesarean section during first stage in nulliparae. Given that the individual has not reached the specific endpoint, a hazard ratio $>1$ at a certain time $t$ indicates an immediately increased relative risk of a transition to the specified endpoint. Estimates are adjusted for confounders, see Table 2 for detailed results. Nulliparae; onset of labour—caesarean section during first stage

\section{Results}

Nulliparae $(n=2,090)$ and parae $(n=1,873)$ were analysed. An intrapartum amniotomy was performed in more than one-third of all nulliparae (34.4\%) and nearly half of all parae (41.8\%, Table 1). Oxytocin augmentation was performed nearly twice as frequently in nulliparae $(52.4 \%)$ as in parae $(27 \%)$. Its median initiation was $6 \mathrm{~h}$ after the onset of labour in nulliparae and $4 \mathrm{~h}$ in parae. The median time from the start of augmentation with oxytocin until birth was shorter in parae (nulliparae median $3.2 \mathrm{~h}$, parae median $1.4 \mathrm{~h}$ ). Neuraxial analgesia was administered in

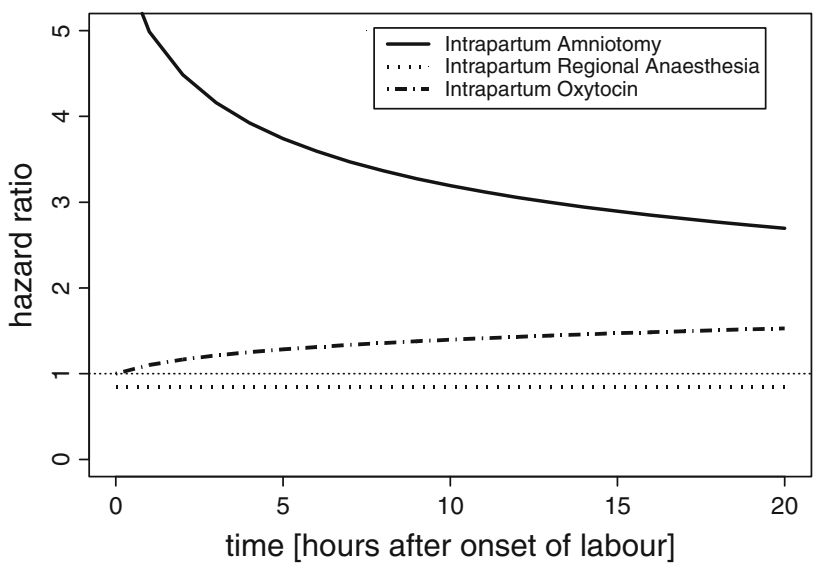

Fig. 3 Plotted graph of the effects of amniotomy, oxytocin and neuraxial analgesia on first stage duration (onset of labour-full dilatation) in parae. Given that the individual has not reached the specific endpoint, a hazard ratio $>1$ at a certain time $t$ indicates an immediately increased relative risk of a transition to the specified endpoint. Estimates are adjusted for confounders, see Table 2 for detailed results

one-third $(34.8 \%)$ of nulliparae and $12.4 \%$ of parae. The median time between onset of labour and neuraxial analgesia was $4.5 \mathrm{~h}$ in nulliparae and $3.2 \mathrm{~h}$ in parae. The median interval between the administration of neuraxial analgesia and birth was $5.2 \mathrm{~h}$ in nulliparae and $2.9 \mathrm{~h}$ in parae.

First-stage factors associated with labour progression and mode of birth

The start of augmentation with oxytocin was associated with acceleration of the progression towards both the predefined endpoints, full dilatation and caesarean section (Figs. 1, 2, 3; Table 2, row 3). For nulliparae, oxytocin augmentation was associated with a permanently higher risk of undergoing a caesarean section, indicated by a hazard ratio of 2.2 (Fig. 2; Table 2, row 7).

The timing of neuraxial analgesia placement was associated with prolonged first-stage labour in parae (Fig. 3; Table 2, row 4). In nulliparae, neuraxial analgesia was associated with a prolonged first stage when administered during the first $7 \mathrm{~h}$ of labour and an accelerated first stage when administered after $7 \mathrm{~h}$ (Fig. 1; Table 2, rows 4, 5). Most importantly, the later neuraxial analgesia was applied in both nulliparae and parae, the more the risk of a caesarean section during first stage increased as compared to those women without neuraxial analgesia (Fig. 2; Table 2, row 9).

The first stage was accelerated when an amniotomy was performed as compared to those women who had already experienced spontaneous rupture of membranes before or after onset of labour, as well as to those whose membranes were still intact (Figs. 1, 3; Table 2, rows 1, 2). However, 


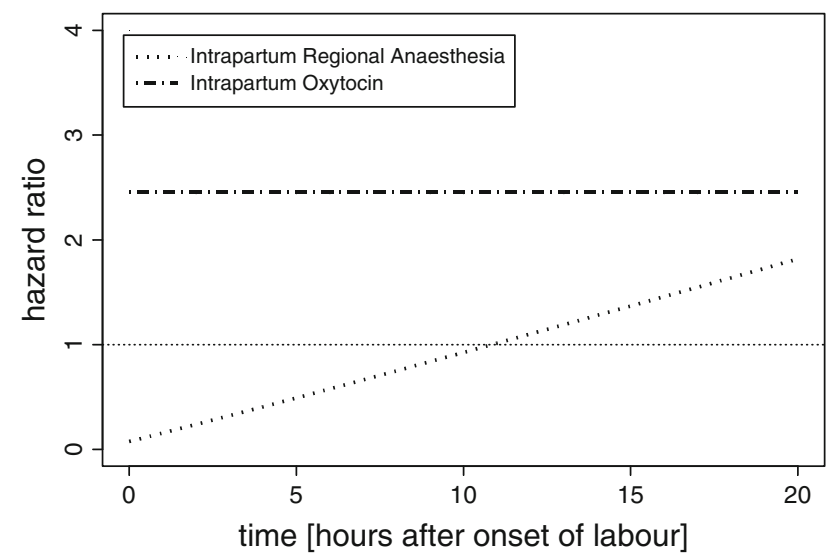

Fig. 4 Plotted graph of the effects of amniotomy, oxytocin and neuraxial analgesia on second stage duration before operative vaginal mode of birth in nulliparae. Given that the individual has not reached the specific endpoint, a hazard ratio $>1$ at a certain time $t$ indicates an immediately increased relative risk of a transition to the specified endpoint. Estimates are adjusted for confounders, see Table 2 for detailed results. Nulliparae, full dilatation—vaginal-operative birth

for nulliparae this observation was accompanied by a constant increase in the hazard ratio to 1.6 for an emergency caesarean section during first stage (Fig. 2; Table 2, row 6). In parae, the initial 6.6-fold acceleration of the first stage declined until the hazard ratio was around 3.5 where the intervention was performed $5 \mathrm{~h}$ after the onset of labour; thereafter, acceleration continued with a hazard ratio of around 3 (Fig. 3; Table 2, rows 1, 2). This means that the degree of acceleration associated with amniotomy varied over time, but that in parae there was some acceleration in the progress of labour throughout its course.

Second-stage factors associated with labour progression and mode of birth

When oxytocin augmentation was applied, increasing risks of an operative vaginal birth in both nulliparae and parae (row 16 in Table 2) and of a caesarean section in nulliparae were observed (row 19). However, the later oxytocin was started in parae, the more likely it was that spontaneous birth would be prolonged (Fig. 5; Table 2, row 12).

Nulliparae with neuraxial analgesia experienced a longer second stage (Table 2, row 13) but a decreased likelihood of operative vaginal birth when the neuraxial analgesia was administered during the first $11 \mathrm{~h}$ of labour (Fig. 4; Table 2, rows 17,18$)$. Conversely, the risk of an operative vaginal birth increased in nulliparae when neuraxial analgesia was administered more than $11 \mathrm{~h}$ after onset of labour (rows 17, 18). In parae, the later neuraxial analgesia was performed, the less likely it was that a spontaneous birth would occur (Fig. 5; Table 2, row 14) and the more likely that a caesarean would be performed (row 20).

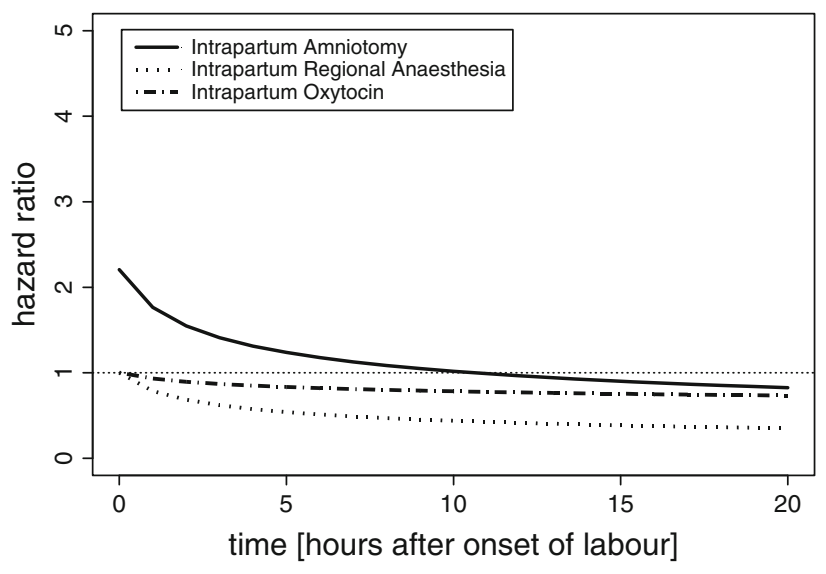

Fig. 5 Plotted graph of the effects of amniotomy, oxytocin and neuraxial analgesia on second stage duration before spontaneous birth in parae. Given that the individual has not reached the specific endpoint, a hazard ratio $>1$ at a certain time $t$ indicates an immediately increased relative risk of a transition to the specified endpoint. Estimates are adjusted for confounders, see Table 2 for detailed results. Parae, full dilatation—-spontaneous birth

Amniotomy increased the tendency for a spontaneous birth in both nulliparae and parae (Table 2, row 10). In parae, the degree of association decreased the later the amniotomy was performed, eventually having the opposite effect after $10 \mathrm{~h}$ (Fig. 5; Table 2, row 11). With parae, the risk of an operative vaginal birth was lower after amniotomy compared to those parae with spontaneous membrane rupture or no amniotomy (row 15).

Time-constant factors during first and second stage

The effects of amniotomy, the start of augmentation with oxytocin infusion and the first administration of neuraxial analgesia were adjusted for time-constant factors (Table 1).

\section{Discussion}

According to our observations, the administration of amniotomy, oxytocin initiation or neuraxial analgesia alters the chance that women receiving them will give birth within a certain time as compared to women not receiving such interventions. The chance concerning birth mode is altered as well. However, these observations need to be interpreted carefully, as interventions are often undertaken due to maternal arrest during labour. Thus, it should be emphasized that we are reporting evidence of time-related associations and not definitive causal relationships between time-dependent interventions and the duration of labour or mode of birth. According to our results, oxytocin initiation is associated with an increased risk of adverse outcomes, 
which could be due to the drug itself [7] or to the confounding effects of an indication. As far as the timing of neuraxial analgesia administration is concerned, in nulliparae prolongation decreases and reverses into acceleration after approximately $7 \mathrm{~h}$. Whether this is due to or at odds with the durational aspect of labour remains unclear.

Quantifying the timing of membrane rupture in relation to the birth process is a new approach for investigating labour duration. Contrary to the findings of a Cochrane review [11], earlier amniotomy results in the earlier achievement of full cervical dilatation in parae. These results demonstrate the need for a randomized controlled trial in which continuously measured amniotomies would be compared with other amniotomies divided into an early and a late group.

As with many interventions, their application is always a question of balancing risk and benefit for the woman and her unborn child. Due to the fact that all women in labour were at risk, irrespective of whether they finally experienced a spontaneous or a non-spontaneous mode of birth, we were able to weigh the risks of selected interventions in relation to alternative endpoints. Cohort studies are able to consider various, but unfortunately not all, factors impacting on labour. Among the limitations of the study are: lack of cervical dilation data, lack of information on uterine contractions, lack of data on foetal head staging, imprecision in defining the onset of labour, lack of data regarding oxytocin dose and titration, lack of information regarding type and drug concentration of neuraxial analgesia, inclusion of VBAC patients, lack of data regarding indications for interventions and non-inclusion of all eligible patients. In addition it has to be taken into account that variables with a significantly altered hazard ratio may not be clinically relevant.

The particular strength of our study lies in the combination of a longitudinal methodological approach with a dynamic environment. This is relevant if interventions have already been proved to be causally related to labour duration and birth outcome. Time-to-event analysis is a promising technique to analyse the timed sequence of interventions during labour [26]. Although our current results are based on a cohort, they have the potential to improve best practise during labour if validated in clinical trials. The degree of association of amniotomy, oxytocin and neuraxial analgesia with the length of the first or second stage of labour changes over time. In some situations, the effect actually reverses direction. These time-related effects should be considered and studied further to enhance the effectiveness of care during labour and improve birth outcomes.

Acknowledgment This study was funded by the German Research Council (DFG).
Conflict of interest None.

\section{References}

1. Chalmers I, Enkin M, Keirse MJNC (1989) Effective care in pregnancy and childbirth. Oxford University Press, Oxford

2. Wang FZ, Shen XF, Guo XR, Peng YZ, Gu XQ (2009) Epidural analgesia in the latent phase of labor and the risk of cesarean delivery. Anesthesiology 111:871-880

3. Wei S, Wo BL, Qi HP, Xu H, Luo ZC, Roy C, Fraser WD (2012) Early amniotomy and early oxytocin for prevention of, or therapy for, delay in first stage spontaneous labour compared with routine care. Cochrane Database Syst Rev 2013(3):CD006794. doi: 10.1002/14651858.CD006794.pub3

4. Wong CA, McCarthy RJ, Sullivan JT, Scavone BM, Gerber SE, Yaghmour EA (2009) Early compared with late neuraxial analgesia in nulliparous labor induction: a randomized controlled trial. Obstet Gynecol 113:1066-1074

5. Ohel G, Gonen R, Vaida S, Barak S, Gaitini L (2006) Early versus late initiation of epidural analgesia in labor: does it increase the risk of cesarean section? A randomized trial. Am J Obstet Gynecol 194:600-605

6. Halpern SH, Abdallah FW (2010) Effect of labor analgesia on labor outcome. Current Opin Anaesthesiol 23:317-322

7. Clark SL, Simpson KR, Knox GE, Garite TJ (2009) Oxytocin: new perspectives on an old drug. Am J Obstet Gynecol 200:35.e1-35.e6

8. Bugg GJ, Siddiqui F, Thornton JG (2011) Oxytocin versus no treatment or delayed treatment for slow progress in the first stage of spontaneous labour. Cochrane Database Syst Rev 2011(7): CD007123. doi:10.1002/14651858.CD007123.pub2

9. Mori R, Tokumasu H, Pledge D, Kenyon S (2011) High dose versus low dose oxytocin for augmentation of delayed labour. Cochrane Database Syst Rev 2011(10): CD007201. doi: 10.1002/14651858.CD007201.pub2

10. Sharma SK, McIntire DD, Wiley J, Leveno KJ (2004) Labor analgesia and cesarean delivery. An individual patient metaanalysis of nulliparous women. Anesthesiology 100:142-148

11. Smyth RMD, Alldred SK, Markham C (2007) Amniotomy for shortening spontaneous labour. Cochrane Database Syst Rev 2007(4):CD006167

12. Petersen A, Ayerle GM, Frömke C, Hecker H, Gross MM (2011) The timing of interventions during labour: descriptive results of a longitudinal study. Midwifery 27:e267-e273. doi:10.1016/ j.midw.2010.10.017

13. Vandenbroucke JP (2004) When are observational studies as credible as randomised trials? Lancet 363:1728-1731

14. Zhang J, Troendle J, Mikolajczyk R, Sundaram R, Beaver J, Fraser W (2010) The natural history of the normal stage of labor. Obstet Gynecol 115:705-710

15. Vahratian A, Troendle JF, Siega-Riez AM, Zhang J (2006) Methodological challenges in studying labour progression in contemporary practice. Paediat Perinat Epidemiol 20:72-78

16. Laughon SK, Branch DW, Beaver J, Zhang J (2012) Changes in labor patterns over 50 years. Am J Obstet Gynecol 206:419.e1-9

17. Neal JL, Lower NK, Ahijevych KL, Patrick TE, Cabbage LA, Corwin EJ (2010) "Active labor" duration and dilation rates among low-risk, nulliparous women with spontaneous labor onset: a systematic review. J Midwifery Wom Health 55:308-318

18. Schiessl B, Janni W, Jundt K, Rammel G, Peschers U, Kainer F (2005) Obstetrical parameters influencing the duration of the second stage of labor. Eur J Obstet Gynecol Reprod Biol 118:17-20 
19. Treacy A, Robson M, O'Herlihy C (2006) Dystocia increases with advancing maternal age. Am J Obstet Gynecol 195:760-763

20. Deutsche Gesellschaft für Gynäkologie und Geburtshilfe e.V. Leitlinien, Empfehlungen, Stellungnahmen (2006) Medikamentöse Wehenhemmung bei drohender Frühgeburt. AWMF 015/025. URL: http://www.dggg.de/leitlinien-2006/pdf-2006/4perinat/4-3-1-med-wehenhemmung.pdf(2007)

21. Gross MM, Wenzlaff P, Haeseler G, Hecker H, Schneider M (2005) Geburtshilflich-anästhesiologische Klinikprofile in Niedersachsen. (Obstetrical-anaesthesiologic clinic profiles in Lower Saxony). 22. Deutscher Kongress für Perinatale Medizin, Berlin 01.-03.12.2005. Z Geburtshilfe Neonatol 209:S104

22. Smith GCS, Pell JP (2001) Use of time to event analysis to estimate the normal duration of human pregnancy. Hum Reprod 16:1497-1500
23. SPSS for Windows (2005) Rel. 14.0.0. SPSS Inc., Chicago

24. Blossfeld HP, Rohwer G (2002) Techniques of event history modeling, 2nd edn. Lawrence Erlbaum Associates, Hillsdale

25. R Development Core Team (2006) R: A language and environment for statistical computing. R Foundation for Statistical Computing, Vienna, Austria. ISBN 3-900051-07-0, URL: http://www.R-project.org

26. Petersen A, Poetter U, Michelsen C, Gross MM (2013) The sequence of intrapartum interventions: a descriptive approach to the cascade of interventions. Arch Gynecol Obstet. doi:10.1007/ s00404-013-2737-8 